\title{
Combining BMI stimulation and mathematical modeling for acute stroke recovery and neural repair
}

\author{
Sara L. Gonzalez Andino ${ }^{1 *}$, Celia Herrera-Rincon ${ }^{2}$, Fivos Panetsos ${ }^{2}$ and Rolando Grave de Peralta \\ ' Electrical Neuroimaging Group, Department of Neurology, Geneva University Hospital, Geneva, Switzerland \\ Neurocomputing and Neurorobotics Research Group, Complutense University of Madrid, Madrid, Spain
}

Edited by:

Niels Birbaumer, Istituto di Ricovero e Cura a Carattere Scientifico Ospedale San Camillo, Italy

\section{Reviewed by:}

Fabio Babiloni, Sapienza University of Rome, Italy

Dennis J. McFarland, Wadsworth

Center for Laboratories and Research, USA

\section{${ }^{*}$ Correspondence:}

Sara L. Gonzalez Andino and Rolando Grave de Peralta, Electrical

Neuroimaging Group, Department of Neurology, Geneva University Hospital, 24 Rue Micheli du Crest, 1211 Geneva 14, Switzerland.

e-mail: sara.gonzalezandino@hcuge.ch; rolando.grave@electrical-neuroimaging. ch
Rehabilitation is a neural plasticity-exploiting approach that forces undamaged neural circuits to undertake the functionality of other circuits damaged by stroke. It aims to partial restoration of the neural functions by circuit remodeling rather than by the regeneration of damaged circuits. The core hypothesis of the present paper is that - in stroke - brain machine interfaces (BMIs) can be designed to target neural repair instead of rehabilitation. To support this hypothesis we first review existing evidence on the role of endogenous or externally applied electric fields on all processes involved in CNS repair. We then describe our own results to illustrate the neuroprotective and neuroregenerative effects of BMl-electrical stimulation on sensory deprivation-related degenerative processes of the CNS. Finally, we discuss three of the crucial issues involved in the design of neural repair-oriented BMIs: when to stimulate, where to stimulate and - the particularly important but unsolved issue of - how to stimulate. We argue that optimal parameters for the electrical stimulation can be determined from studying and modeling the dynamics of the electric fields that naturally emerge at the central and peripheral nervous system during spontaneous healing in both, experimental animals and human patients. We conclude that a closed-loop BMI that defines the optimal stimulation parameters from a priori developed experimental models of the dynamics of spontaneous repair and the on-line monitoring of neural activity might place BMls as an alternative or complement to stem-cell transplantation or pharmacological approaches, intensively pursued nowadays.

Keywords: neuroprotection, plasticity, neuroprostheses, rat, trigeminal, peripheral nerve

\section{INTRODUCTION}

Ischemic stroke is the major cause of adult disability (including paralysis) and cognitive deficits (Burns et al., 2009) and the third main cause of death after heart disease and cancer. While approximately $75 \%$ people survive their initial stroke, just onethird of them recover completely. According to the World Health Organization (2007) 15 million people suffer stroke worldwide each year. Of these, five million die and another five million remain permanently disabled. This long-term disability implies that most of the costs of stroke are incurred in supporting stroke survivors whose number is expected to increase due to the natural aging of the population.

Although stroke damage can be devastating, some patients survive the initial event and undergo a certain level of spontaneous recovery that can be further augmented by rehabilitative therapies (Murphy and Corbett, 2009; O'Dell et al., 2009; Johansson, 2011). Rehabilitation exploits neural plasticity to adapt the undamaged neural circuits to undertake the functionality of the circuits damaged by stroke (Johansson, 2011). Neurorehabilitation aims to partial restoration of the neural functions by circuit remodeling rather than by the regeneration of damaged circuits. It is based on the observation that cortical representation areas and cortical maps, can be modified by sensory input, experience, and learning. However, while the juvenile brain exhibits a high capacity for plasticity and repair, this capacity considerably declines with age whereas the risk for stroke increases (Popa-Wagner et al., 2007). Rehabilitation usually begins immediately in the hospital once a patient is medically stable, and can continue on for months to years depending on the severity of the disability. Complete rehabilitation becomes an arduous, expensive and often impossible task as shown by the high proportion of patients that remain permanently disabled.

Brain machine interfaces (BMIs) are gaining increasing importance within stroke rehabilitation (Popovic et al., 2009). Current research and proposed devices are focusing within three major lines of application: (1) Improving the rehabilitation outcome trough functional electrical stimulation (FES) of limbs (Barreca et al., 2003) and or spinal cord that helps to regain mobility (Yan et al., 2005), stabilize gait (Kesar et al., 2009), or regulate bladder and bowel functionality (Peckham and Knutson, 2005); (2) Retraining reaching and grasping functions (Popovic et al., 2005), and (3) Transforming neural commands representing subjects intentions into effective motor commands transmitted to robotic arms or other prostheses as reviewed in (Chae and Hart, 2003; Birbaumer and Cohen, 2007; Mak and Wolpaw, 2009).

Due to the difficulties inherent to rehabilitation in the elderly and the relatively low success rate of current techniques, research in stroke is gradually moving into the direction of neural repair (Murphy and Corbett, 2009; Carmichael, 2010). This is a new trend supported by relatively new experimental results (Gross, 2000) showing that the mature nervous system is able to create new neurons (neurogenesis), and that newly formed neurons are able to grow (sprouting) and extend their axons to form new synapsis (synaptogenesis). 
Within neural repair, one of the major trends is the grafting or injection of multiple types of stem-cells to enhance regeneration and functional recovery (Borlongan, 2009; Carmichael, 2010). However, for the moment and compared to the initial expectatives, stem-cell-based therapy has reached a relatively modest level of success in clinical trials (Kondziolka et al., 2005; Banerjee et al., 2010). Indeed, it is not yet clear if the observed functional improvements are due to a differentiation of the stem-cells in neurons and their integration into the injured CNS or their presence simply provokes secretion of grow factors that contribute to the regeneration of the tissues (Murphy and Corbett, 2009).

A different approach, today considered as the most promising one for the future of stroke patients, is to directly act on the causal molecular mechanisms that control tissue regeneration and on the design of pharmacological approaches to manage them (Carmichael et al., 2005; Benowitz and Carmichael, 2010). It has been recently proposed that a good model to start searching for causal mechanisms in tissue regeneration is the spontaneous or self-healing process that occasionally takes place after stroke in selected groups of patients or animals (Carmichael, 2006; Murphy and Corbett, 2009). Animal studies indicate that genes and proteins important for the three key aspects in neural repair, namely neural growth, synaptogenesis, and neural sprouting, expressed at their highest levels during early brain development (Hattiangady et al., 2005) are also expressed during a short-lived period that opens immediately after stroke (Murphy and Corbett, 2009). Consequently it is hoped that the understanding of the natural recovery mechanisms will help us in the development of therapeutic processes to augment and/or accelerate the self-repair process.

The core hypothesis of the present paper is that - in stroke - BMIs can be designed to target neural repair instead of rehabilitation. To support this hypothesis we first review existing evidence on the role of endogenous or externally applied electric fields on all processes involved in CNS repair. We then describe our own results to illustrate the neuroprotective and neuroregenerative effects of BMI-electrical stimulation on sensory deprivation-related degenerative processes of the CNS. Finally, we discuss three of the crucial issues involved in the design of neural repair-oriented BMIs: when to stimulate, where to stimulate and - the particularly important but unsolved issue of - how to stimulate.

With respect to the use of mathematical models we argue that optimal parameters for the electrical stimulation can be determined from studying and modeling the dynamics of the electric fields that naturally emerge at the central and peripheral nervous system during spontaneous healing in both, experimental animals and human patients.

We conclude that a closed-loop BMI that defines the optimal stimulation parameters from a priori developed experimental models of the dynamics of spontaneous repair and the on-line monitoring of neural activity might place BMIs as an alternative or complement to stem-cell transplantation or pharmacological approaches, intensively pursued nowadays.

\section{THE ROLE OF NATURALLY GENERATED ELECTRICAL FIELDS IN NEURAL REPAIR, PLASTICITY, AND NEUROGENESIS}

In this section we briefly review some of the evidences that support our hypothesis that neural repair could be artificially induced through an appropriate modulation of intracellular and extracellular fields by means of BMIs.

\section{STROKE DAMAGE AND MECHANISMS FOR NATURAL RECOVERY SUITABLE TO BE PROMOTED BY BMI STIMULATION}

Around one-third of stroke patients survive the initial event and undergo partial or complete spontaneous recovery (Murphy and Corbett, 2009; Johansson, 2011). In the initial stage of the recovery (weeks 1-4) different homeostatic mechanisms appear that contribute to the re-activation of the stroke-affected areas through structural and functional changes of the neural circuits.

Stroke-affected tissue is usually divided into a core area, characterized by complete blood deprivation and cells' death, and a periinfarct area, termed the penumbra, characterized by reduced blood flow and damage of the neural cells and neuropil (axon terminals, dendrites, and glia). In animal models of focal stroke, diaschisis a sudden loss of function in a portion of the brain connected to but at a distance of a damaged area - is also observed in regions adjacent and connected to the core (Carmichael et al., 2004). While at the core there is a severe and rapid tissue injury, brain cell death evolves more slowly within the penumbra zone (Lo et al., 2005). In acute ischemic stroke in primates the neurons at the penumbra remain structurally intact but functionally inactive (Astrup et al., 1977). Without reperfusion, i.e., restoring nutritional blood flow to threatened tissues before they progress to infarction (Molina and Saver, 2005), the penumbra collapses, brain cells die, and the lesion expands (Lo et al., 2005).

Functional recovery is thought to depend on function remapping from the core to the penumbra area. Indeed, saving of the penumbra has been shown to have definite and predictable benefit on subsequent neurological recovery in man (Guadagno et al., 2003). Recovery of the penumbra impacts both, early and late recovery (Furlan et al., 1996). Remapping depends on reperfusion and on latent subthreshold inputs to the penumbra region combined with a rewiring of the neural connections promoted by axonal sprouting and synaptogenesis (Murphy and Corbett, 2009). Both, remapping and rewiring are promoted by Hebbian-like, activity-dependent, synapse-based learning rules that strengthen and refine the circuits if (and only if) there is some circuitry to route sensory signals to the brain and motor commands out of it (Murphy and Corbett, 2009). Importantly, while the mature brain is able to create new neurons (neurogenesis) this effect is limited to selected brain areas. However, new neurons can migrate to the peri-infarct zone. The necessary condition to facilitate the restoration of circuit activity over days to weeks through compensatory rewiring or remapping seems to be the presence of some of the original connections to provide weak sensory and motor signals, subsequently enhanced through neural plasticity. Rerouting and rewiring for saving the penumbra should also lead to prevention of the diaschisis.

In summary, functional recovery after stroke seems to heavily depend on:

- Saving penumbra cells from death: This is dependent on early reperfusion further promoted by angiogenesis (the growth of new capillary vessels) as well as by insuring sufficient synaptic input to the penumbra area.

- Promoting rewiring and remapping in the penumbra by stimulating synaptogenesis and axonal sprouting. Axonal sprouting (rewiring) and functional remapping are based on 
synapse-based learning rules that depends on the existence of synaptic input and the circuitry to route sensory signals to the brain and motor commands out of it.

- Preventing diaschisis. If the circuitry to route sensory signals to the brain and motor commands out of it is altered due to the loss of function in areas connected to the core, synaptic input is interrupted. As mentioned before, synaptic input to the penumbra is essential for rewiring, remapping, angiogenesis, and preventing cellular death.

A BMI aiming to neural repair should target the above mentioned goals rather different from those pursued nowadays in BMI-based stroke rehabilitation. However, to be effective in these directions, BMIs should be capable to effectively modulate electric fields on the CNS given their beneficial effects on neural repair described next.

\section{ELECTRIC FIELDS INFLUENCE ANGIOGENESIS, NEUROGENESIS, AND SYNAPTOGENESIS \\ Spontaneously emerging intrinsic electric fields}

As Maxwell equations predict but for too long ignored in Neuroscience, neurons apart from generating electromagnetic fields are also sensitive to them. There is experimental evidence accumulated over the last 5 years supporting that the weak electric fields generated in a natural way by the physiological activity of neocortical networks are strong enough to (i) significantly modify the excitability of their own cells and (ii) alter the synchronization of the networks themselves (Fröhlich and McCormick, 2010; Mann and Paulsen, 2010; Weiss and Faber, 2010). Moreover, electric fields and biochemical neural processes are deeply intermingled and, therefore, electric fields are likely to also affect dendritic conductances, transmitter release, and the diffusion of ions and charged neurotransmitters (Weiss and Faber, 2010).

Altering excitability and network synchronization are keys to the two synaptic learning rules, known to influence CNS synaptogenesis and plasticity (Murphy and Corbett, 2009): (1) Hebbian plasticity mechanisms that favor the rewiring of neural pathways and (2) homeostatic plasticity mechanisms that ensure that neurons receive an adequate amount of synaptic input. It has been proved that self-repair processes are accompanied by the spontaneous modulation of extracellular fields (Carmichael and Chesselet, 2002; Carmichael, 2003; McCaig et al., 2009; Floel and Cohen, 2010) and the emergence of synchronous activity (Carmichael and Chesselet, 2002). Moreover, the spontaneously appearing synchronous neuronal activity is a signal for axonal sprouting after cortical lesions (Carmichael and Chesselet, 2002).

The appearance of electric fields is not a prerogative of the central nervous system. Direct-current (DC) electric fields naturally arise in all developing and regenerating animal tissues although their existence and potential impact on tissue repair and development remain largely unexplored (McCaig et al., 2005, 2009). Cells undergo a variety of physiological processes including division, migration, and differentiation, under the influence of endogenous electrical cues which are generated physiologically or pathologically in the extracellular and sometimes intracellular space. In almost all systems that have been studied, crucial behaviors such as cell division, cell migration, and cell differentiation take place within an extracellular milieu in which standing voltage gradients persist for several hours or even days (McCaig et al., 2005, 2009).

\section{THE ROLE OF ARTIFICIALLY INDUCED ELECTRIC FIELDS IN NEURAL REPAIR, PLASTICITY, AND NEUROGENESIS}

In ischemic rats, direct invasive electrical stimulation of the cerebral cortex has been shown to exert antiapoptotic, angiogenic, and anti-inflammatory effects (Baba et al., 2009). Electrical stimulation seems to render the ischemic brain as a nurturing microenvironment characterized by robust angiogenesis and diminished microglial/astrocytic proliferation, resulting in the reduction of infarct volumes and promoting behavioral recovery (Baba et al., 2009). The repairing role of peripheral stimulation (further illustrated in the next section) is also crucial: manual stimulation of the whiskers in the ischemic rat promotes angiogenesis and enhances blood flow in the penumbra (Whitaker et al., 2006), enhances neurogenesis, and neural progenitor migration toward the ischemic barrel cortex (Li et al., 2008) and increases the vascular endothelial growth factors (Whitaker et al., 2006). This happens because manual stimulation of the whiskers excites the mechanoreceptors located under the skin which transform these mechanical stimuli to electrical pulses that travel to the central nervous system through the peripheral nerves.

Consequently, physiologically weak electrical fields and ionic currents should be the electrical equivalents to cellular therapies for neural repair, axonal guidance, and chemotropism given their role on neurogenesis, nerve repair, promotion, and orientation of axonal sprouting and even on cell migration in damaged neural tissues (Yao et al., 2008, 2009; Sundelacruz et al., 2009).

\section{NEUROPROTECTIVE EFFECTS INDUCED BY BMI STIMULATION}

In this section, we provide evidence that neuroprotective and neuroregenerative effects similar to those provoked by manual stimulation of the whiskers (Angiogenesis, neurogenesis, and migration of progenitor cells toward the ischemic areas) can be achieved by direct electrical stimulation of the peripheral nerves through an implanted BMI.

Indeed, peripheral and central nervous system are completely interrelated. Since Merzenich et al. (1983) proved that transection of a peripheral nerve modifies the cortical representation of the injured limb, many studies have focused on structural and physiological alterations in deafferented central pathways (for reviews see Merzenich et al., 1990; Jain et al., 1998; Kaas, 2000; Blake et al., 2002). Changes in neural activity can be quantified in several ways (histologically, electrophysiologically, etc.). Particularly useful is quantification using cytochrome oxidase (CyO), a critical enzyme whose levels correlate with chronic changes of the synaptic activity. An increase of the sensory stimulation or behavioral training provokes an increase in the energetic demand of the sensory areas and, consequently, an increase in the expression of $\mathrm{CyO}$ while a decrease of the sensory stimulation provokes the opposite effect (WongRiley, 1989; Bruchey and Gonzalez-Lima, 2008). Also Parvalbumin (PV) and Calbindin (Calb), two calcium-binding proteins (CaBPs) present in cortical interneurons, are indicative of the modifications of the neural activity and their expression levels are altered after peripheral manipulations. 
In determining the effects of peripheral manipulations to the central nervous system the trigeminal somatosensory system of the rat has resulted particularly useful due to its highly topographic organization (Waite and Tracey, 1995; Diamond et al., 2008; Panetsos and Sanchez-Jimenez, 2010). This is because in all relay stations information processing is done by groups of neurons spatially distributed in the same manner as the whiskers on the snout on the animal (somatotopic maps) and each group of neurons receives its main input from only one whisker in the periphery. In cortical layer IV these groups of neurons take the shape of barrels forming the cortical "barrel field" (Figure 1, see also Woolsey et al., 1975).

To assess the restoring effects of BMIs on damaged cortical neural networks we compared the barrel cortices of rats with transaction of the peripheral nerve (and a consequent massive deafferentation of the cortex, lesioned, or LES-animals) and the barrel cortices of animals with the same manipulation but to which a stimulating device was implanted immediately after the lesion (whose cortices received artificially generated currents from the BMI, BMI-animals). Electrical stimulation was delivered to the proximal stump of the transected nerve for 4 weeks, $12 \mathrm{~h} /$ day, using $100 \mu$ s-long pulses, $3.0 \mathrm{~V}$, and with a frequency of $20 \mathrm{~Hz}$. Animal handling, housing, surgery, and euthanasia were carried out according to current national legislation (R.D. 223/88), and EU Directives (86/609/EC) on this matter. In Figure 1 a schematic presentation of the experiment can be shown. More details on the experimental procedures are given in (Herrera-Rincon et al., 2010a,b).
Effects induced by BMI stimulation were quantified at all levels of the trigeminal sensory pathway, i.e., we estimated the metabolically active volume of whisker-related regions in brainstem, thalamus, and cortex and we estimated the number of cortical interneurons expressing CaBPs in LES- and BMI-animals. In every animal we compared the two hemispheres, manipulated versus control (experimentally affected versus intact). After 4 weeks LES-animals showed a severe decrease in the cortical activity and a reduction in the number of PV- and Calb-positive neurons (Figure 2A) while no BMI-animal displayed differences between control and experimental hemispheres (Figure 2B). Similar results were observed in the lower stations of the somatosensory pathway.

Our results confirm that loss of sensory input from the peripheral nervous system leads to structural and functional changes at the barrel cortex and that such changes can be avoided by the very early use of BMI. Indeed, these results show that chronic electrical stimulation directly applied to the proximal stump of the transected nerve can be used to prevent the significant loss of synaptic activity in the cortex and distant interconnected structures. BMI stimulation displayed a clear neuroprotective and neuroregenerative effect to the affected regions.

\section{WHEN, WHERE, AND HOW TO STIMULATE WITH BMIS TO OPTIMALLY PROMOTE NEURAL REPAIR}

In the present paper we defend the idea that, in stroke, functional recovery can be effectively obtained by using BMI stimulation, alone or in combination with other therapeutic techniques. In

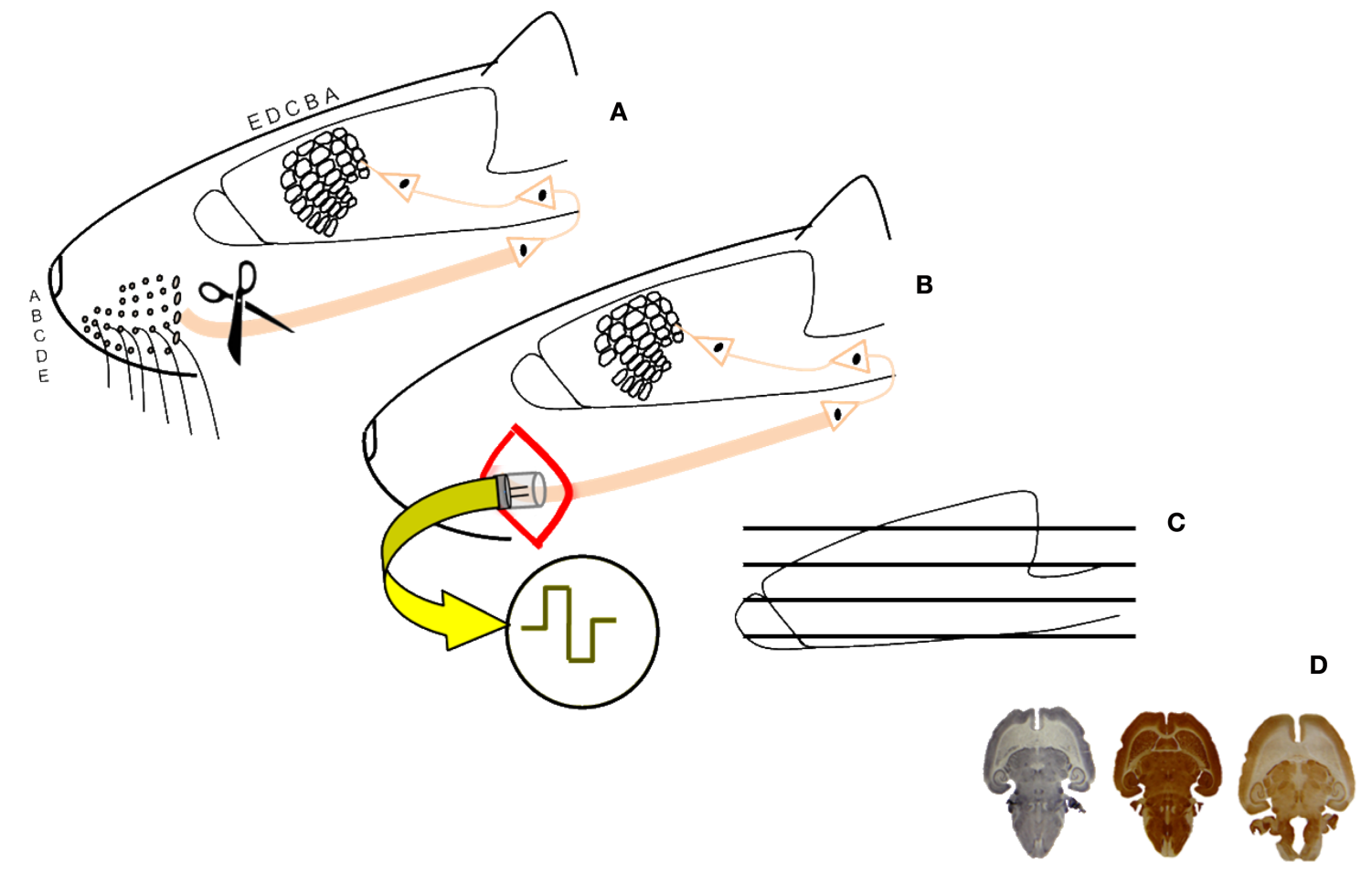

FIGURE 1 | Schematic diagram of the experimental procedure. Unilateral section of the sensory trigeminal nerve (A); implant of a stimulation device in half of the animals and 4-weeks artificial stimulation of the nervous system (B); sections and histochemistry (C) histological, stereological study of the brains (D). 

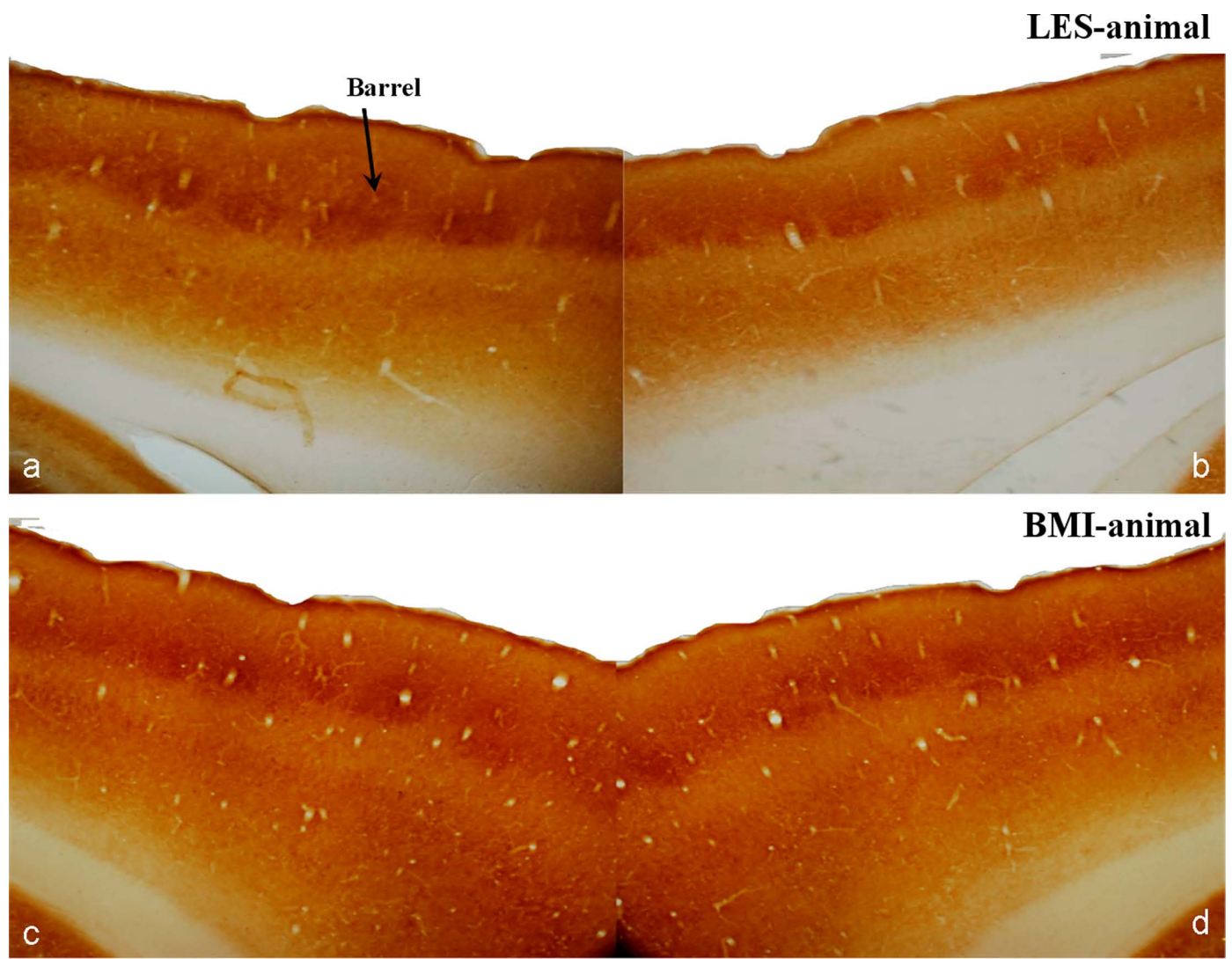

CONTROL HEMISPHERE

FIGURE 2 | Photomicrographs of cytochrome oxidase (CyO)-stained horizontal sections of rat somatosensory cortices at the level of the barrel field. They correspond to animals with unilateral lesions of the trigeminal nerve [input-deprivation of the contralateral somatosensory cortex-sections in $(\mathbf{a}, \mathbf{c})$ ] and animals with identical lesions but with subsequent artificial stimulation by means of an external device [BMI input to the contralateral cortex - sections in (b,d)]. Brain hemispheres receiving input from non-manipulated nerves (control)

\section{EXPERIMENTAL HEMISPHERE}

the previous section we justified our approach using both, the most recent bibliography in this field and experimental data from our labs. However, the mere proposal of a novel approach or technique, although well documented and justified, is not sufficient for future applications or even research if it is not accompanied by precise indications on how to use it. For example electrical stimulation has been used for a while to treat the symptoms in Parkinson disease. Nevertheless, the basic functional mechanisms and, more particularly the optimal stimulation parameters that could allow us to reach a full recovery of the damaged tissues, remain largely unknown the application of BMIs to the treatment of this disorder being far limited by the scarce information available on these issues (Logothetis et al., 2010).

In the present section we describe the way our theory could be tested and, if correct, to be applied for neural repair in stroke patients. We consider that all necessary information is available in the natural healing processes and can be extracted by means of precise experiments and mathematical models. are left and experimentally affected are right. Asterisk in [a] points to a single barrel of the fourth cortical layer. Animals with nerve lesions show a lower intensity of CyO-staining in the affected hemisphere [b] indicating the lower activity of the affected somatosensory cortex in comparison to the control one [b]. Artificial stimulation has a net positive effect to the affected cortex and maintains the neural activity to a normal level as proved by the similar staining intensities in the cortexes of BMl-animals [b,d]. Original magnifications $20 \times$.

\section{WHEN BMI STIMULATION MUST BE APPLIED? CRITICAL TIME-WINDOW FOR OPTIMAL RESULTS}

Brain machine interfaces should be introduced in the acute/subacute period after stroke to exploit and widen the Critical Phase, a spontaneously opening time-window of neural plasticity occurring approximately between the 5 th and 20 th-day.

Self-repair processes such as self-induced neuroregeneration and self-induced neuroprotection emerge during this Critical Phase. Genes and proteins important for neural repair, the same that are expressed during early brain development, where there is maximal neural growth, neural sprouting, and synaptogenesis, are also expressed at very high levels (Murphy and Corbett, 2009) immediately after stroke. It is during this phase that damaged cells, still alive in the penumbra area, have to be protected and maintained alive. By the end of the Critical Phase is marked by the extinction of the self-repair processes, and the closing of the neuroplasticity window. At this point neural repair becomes impossible and rehabilitation techniques can be introduced to facilitate functional recovery but not neural repair! Indeed, neurorehabilitation fails to exploit both, 
the self-repair mechanisms and the preserved functional specialization of the partially undamaged neural circuits, so functional recovery is considerably delayed and hardly ever complete.

We propose that this Critical Phase of heightened neuroplasticity must be both, investigated and exploited to initiate any treatment aimed to neural repair. As the only period during which self-repair mechanisms and structurally damaged, but functionally specialized neural circuits, co-exist, the Critical Phase is the optimal and probably the only period to artificially induce neural repair. Moreover, as shown before, central, or peripheral electrical stimulation exert angiogenic effects and promotes vascular growth factors preserving the functionality of individual neurons and entire regions then it can be potentially used to extend the Critical Phase, i.e., to extend the time limited period of neural survival keeping the optimal conditions for neural repair.

\section{WHERE BMI STIMULATION MUST BE APPLIED? STIMULATION OF THE CENTRAL AND PERIPHERAL NERVOUS SYSTEM}

Stroke damages are not limited to the core or the penumbra area but they can reach distant regions physically connected to the core of the stroke by means of neural pathways. In addition, loss of a limb or it's prolonged immobility leads to neurodegenerative processes in the whole somatosensory pathway and in particular in the cortex as a result of the chronic deafferentation/reduction of sensory stimuli and the subsequent drop of direct synaptic input. Consequently, BMI stimulation must be applied in such a way to influence both, the penumbra area and the neural pathways that interconnect the affected regions in the brain. On one hand we have to control the electric fields in the penumbra area to locally promote synaptogenesis and angiogenesis. On the other hand we have to maintain functional all the circuitry that routes sensory signals into the brain and motor signals outside from it to allow Hebbian plasticity to be effective.

As seen before, both, central and peripheral electrical stimulation exert neuroprotective and neurorepair effects in animal models of stroke. To take advantage from both techniques and also to increase their effectiveness in a synergistic approach neural repair should rely on both

- direct brain stimulation to dynamically modulate electric fields in the extracellular space that will influence the extracellular matrix and therefore neurogenesis/sprouting and homeostatic plasticity and

- peripheral stimulation expected to basically impact Hebbian and homeostatic plasticity.

Controlling the dynamic of electric fields at the penumbra area can be achieved by both, invasive and non-invasive BMIs. Neural implants at the penumbra can provide a finely tuned control of extracellular fields but the risk of surgical intervention at the acute and post-acute stages of stroke needs to be carefully weighted. Noninvasive BMIs in the form of central stimulation can be based on transcranial magnetic stimulation (TMS) or transcranial directcurrent stimulation (tDCS).

Brain machine interface-originated electric fields must lead to a functional re-activation of the sensory pathway, hence to maintain the organization of the neural circuits by means of the aforemen- tioned Hebbian rule. Such effects will be mediated by the action of the electrical stimulation on the activity-depending signaling pathways, for example by promoting genes transcription, necessary for synaptic transmission or long-term potentiation (Tan et al., 2008). This way BMIs should contribute to the creation and maintenance of a molecular microenvironment similar to the one that spontaneously arise after stroke in cases of successful self-repair.

The above considerations on the maintenance of functional pathways and the importance of a high neural activity toward and from the affected regions make clear that, due to its neurostimulating nature, neurorehabilitation should complement the BMI approach if we want to achieve real/effective functional restoration after neural repair.

\section{HOW BMI STIMULATION MUST BE APPLIED? THE NEED OF MATHEMATICAL MODELS}

The effectiveness of BMI stimulation, as that of every clinical treatment, depends on the knowledge we have on the specific disease and the mechanisms of action of the treatment that define the therapeutic protocol. Unfortunately, while invasive and non-invasive devices for FES are widely used for other neurological diseases (e.g., Parkinson) our limited knowledge hinders the exploitation of the enormous potential of these methods. Indeed most stimulating BMIs rely on fixed stimulation protocols where the stimulation frequency and intensity do not vary over time.

Contrarily to other diseases of the CNS, in stroke, the existence of naturally occurring self-repair processes allows us to determine stimulation procedures for optimal BMI-based neural repair. It is plausible that the same temporal dynamics followed by the electric fields in case of successful spontaneous healing serve to artificially promote neural repair where self-repair does not spontaneously arise or even to increase the effectiveness of the spontaneously arising healing processes. By modeling such processes and then by studying the behavior of the electric fields we will be able to determine the correct way to stimulate and establish the ranges of the parameters to obtain optimal neural repair by means of BMIs. Accordingly, it is necessary to investigate the relationship between the dynamics of spontaneously emerging electrodynamic fields and the self-induced repair processes that lead to a complete recovery of the central nervous system using data from both, animal models and human patients.

The development of mathematical models for the healing processes in stroke can rely on both, experimental data from in vivo animal models in rodents and human clinical data from human patients. Information theory and its close cousin Bayesian modeling provides an ideal framework to uncover hidden dependencies between the temporal evolution of healing and the dynamics of spontaneously generated electric fields as these models assume no fixed (e.g., linear) physical relationships between the variables. Rather the dependencies are discovered from repetitions of the observations.

\section{MATHEMATICAL MODELING OF HEALING PROCESSES AFTER STROKE}

In animals, we can rely on extracellular measurements of local field potential (LFPs) and single/multi unit activity (SUA/MUA) on one hand and the anatomical-structural changes in the neural tissue on the other to establish specific links between the naturally occurring electric fields and functional/structural recovery. The link between electric field dynamics and neural recovery can be established by 
correlating specific changes in the electrophysiological recordings with the diverse histochemical measures of network activity and connectivity: amplitude, frequency, hyperexcitability, synchrony, and different functional connectivity measures in the first case and metabolic activity, number of neurons, axonal sprouting, neurogenesis, etc., in the second.

In humans, scalp EEG can be monitored during the acute/postacute phase of stroke and after the transfer of patients to the rehabilitation unit. As scalp EEG lacks the spatial resolution to assess the properties of ongoing phenomena within the core/penumbra area one could rely on the non-invasive estimation of field potentials or currents (Grave de Peralta Menendez et al., 2000). With such approach we should be able to correlate frequency specific changes in the core/penumbra with structural/functional and metabolical changes detected from non-invasive neuroimaging techniques such as MRI/PET/fMRI. Furthermore, frequency specific changes in the estimated signals at the core/penumbra can be correlated with electrophysiological measures of local network connectivity.

All data can be obtained under electrodynamic stimulation to characterize electric field dynamics during the acute phase to identify common features of spontaneous recovery. The idea is to establish unambiguous links between electrodynamic fields at the penumbra area and the level of functional and structural recovery derived from specific circuit changes or behavior using information theory mathematical tools.

The use of experimental animal models of stroke in rodents provides crucial information that is impossible to obtain from human subjects:

- Electrophysiological characterization of electrodynamic phenomena within the core and the penumbra area to create the information theoretical models that can guide BMIs to induce optimal repair and recovery.

- Fine quantitative assessment of the effects of artificially induced electrodynamic fields on the neural repair via the electrophysiological (Hebbian and homeostatic plasticity) and histochemical analysis of the neural tissue (metabolic activity, number of neurons, connectivity, axonal sprouting, neurogenesis, etc.).

- Functional assessment of the recovery in terms of behavioral markers.

Rodent models of stroke allow correlating spatially tuned frequency specific changes in the measured fields with the diverse histochemical and electrophysiological measures of the recovery of the neural circuits. It is from these correlations that appropriate models for stimulation that mimic the sequence of natural recovery can be devised. However, realistic models in rodents should be relatively small as survivable human strokes affect $5-15 \%$ of the hemisphere. Examples of well established models of small strokes in rodents are the Middle cerebral embolism and the Photothrombosis (Murphy and Corbett, 2009) which bears the additional advantage of being non-invasive. Moreover, rodent models permit the a posteriori assessment of the effectiveness of invasive and non-invasive stimulation directly applied to the penumbra area and the comparison with the less invasive peripheral stimulation if applied alone.
The use of information theory provides an ideal framework to:

- Characterize the dynamics of self-repair processes using concepts such as entropy or mutual information.

- Evaluate which parameters of spontaneous electrodynamic fields influence self-repair, as measured by conventional electrophysiological, histochemical, neuroimaging, or behavioral techniques.

- Model the recovery process, for example as Markov stochastic processes.

- Determine the optimal dynamic for the implementation of BMI devices oriented to artificially promote neural repair.

The self-healing process can be initially modeled as a Markov stochastic process to relate the sequences of measured electrodynamic events to the transitions in the state of the system. Such model could be able to dynamically determine the parameters that determine optimal state transitions (i.e., those that were previously learned as leading to successful self-repair in the animal and human models). Note that the dynamical evaluation of entropy rates during spontaneous and artificial healing could constitute a new clinical prognostic measure of the possibility to repair the nervous system after its acute damage.

Once the characterization of the optimal dynamic changes of the fields is established we will be able to create more advanced models describing the sequence of the stimulating parameters as a function of the state of neural networks inferred from the real time monitoring of the brain electrical activity. This would therefore constitute a closed-loop BMI that defines the optimal stimulation parameters from the on-line monitoring of neural activity and the existing model of the dynamics of neural repair.

\section{CONCLUSIONS}

In the present paper we provide theoretical and experimental evidence that conveniently designed BMIs could be used to achieve neural repair in stroke patients. BMI-based stroke therapies, alone or combined with other advanced approaches (e.g., stem-cell therapies) could go far beyond the limits of up-to-day clinical treatments for stroke but in any case, to be effective, they should be followed by neurorehabilitation techniques (classical, virtual, etc.).

Theoretical evidences were given to sustain that (i) natural activity of the neural networks can generate electric fields of sufficient strength to influence themselves (ii) low intensity electric fields arise spontaneously in a short time-period after stroke in the damaged area and (iii) these spontaneously arising low intensity electric fields play a very important role in the effectiveness of after stroke self-repair mechanisms. On this basis, we suggest that it should be equally possible to artificially reproduce (using electrical stimulation) the electrical conditions measured in vivo in the extracellular space during the processes of natural healing.

Experimental evidences were presented to support that (i) BMIs can preserve the functionality of brain areas deprived from natural sensory input and (ii) can be used to influence the two plasticity rules that play a crucial role in neural repair and recovery processes: homeostatic plasticity that ensure that neurons receive an adequate 
amount of synaptic input and Hebbian plasticity that redistribute synaptic strength to favor the wiring of pathways that are coincidently active.

The aforementioned evidences suggest that by correctly modulating ionic currents and extracellular electric fields via BMIs we should be able to provoke and guide plastic changes of the CNS.

To achieve this ambitious objective we propose to develop information based mathematical models of natural healing processes that help us to determine the optimal stimulation parameters to induce neural repair by means of a closed-loop BMIs that rely on the on-line monitoring of electrical activity.

Besides, selective electrical stimulation of peripheral nerves innervating the core area affected by stroke should be initiated as soon as the location and extent of the penumbra area has been identified from MRI to increase the chances of neural survival and maximally exploit the Critical Phase. In this way we expect that BMIs can become a novel avenue of research that optimally exploit neural repair and plasticity for improving functional and structural recovery after severe ischemic stroke

\section{REFERENCES}

Astrup, J., Symon, L., Branston, N., and Lassen, N. (1977). Cortical evoked potential and extracellular $\mathrm{K}+$ and $\mathrm{H}+$ at critical levels of brain ischemia. Stroke 8, 51-57.

Baba, T., Kameda, M., Yasuhara, T., Morimoto, T., Kondo, A., Shingo, T., Tajiri, N., Wang, F., Miyoshi, Y., Borlongan, C. V., Matsumae, M., and Date, I. (2009). Electrical Stimulation of the cerebral cortex exerts antiapoptotic, angiogenic, and anti-inflammatory effects in ischemic stroke rats through phosphoinositide 3-kinase/Akt signaling pathway. Stroke 40, e598-e605.

Banerjee, S., Williamson, D., Habib, N., Gordon, M., and Chataway, J. (2010). Human stem cell therapy in ischaemic stroke: a review. Age Ageing 40, 7-13.

Barreca, S., Wolf, S. L., Fasoli, S., and Bohannon, R. (2003). Treatment interventions for the paretic upper limb of stroke survivors: a critical review. Neurorehabil. Neural. Repair 17, 220-226.

Benowitz, L. I., and Carmichael, S. T. (2010). Promoting axonal rewiring to improve outcome after stroke. Neurobiol. Dis. 37, 259-266.

Birbaumer, N., and Cohen, L. (2007). Brain-computer interfaces: communication and restoration of movement in paralysis. J. Physiol. (Lond.) 579, 621-636.

Blake, D. T., Byl, N. N., Bedenbaugh, P., Nagarajan, S., and Merzenich, M. (2002). Sensory representation abnormalities that parallel focal hand dystonia in a primate model. Somatosens. Mot. Res. 19, 347-357.

Borlongan, C. V. (2009). Cell therapy for stroke: remaining issues to address before embarking on clinical trials. Stroke 40, S146-S148.

Bruchey, A. K., and Gonzalez-Lima, F. (2008). Enhanced metabolic capacity of the frontal cerebral cortex after Pavlovian conditioning. Neuroscience 152, 299-307.

Burns, T. C., Verfaillie, C. M., and Low, W. C. (2009). Stem cells for ischemic brain injury: a critical review. J. Comp. Neurol. 515, 125-144.

Carmichael, S. T. (2003). Plasticity of cortical projections after stroke. Neuroscientist 9, 64-75.

Carmichael, S. T. (2006). Cellular and molecular mechanisms of neural repair after stroke: making waves. Ann. Neurol. 59, 735-742.

Carmichael, S. T. (2010). Targets for neural repair therapies after stroke. Stroke 41, S124-S126.

Carmichael, S. T., Archibeque, I., Luke, L., Nolan, T., Momiy, J., and Li, S. (2005). Growth-associated gene expression after stroke: evidence for a growthpromoting region in peri-infarct cortex. Exp. Neurol. 193, 291-311.

Carmichael, S. T., and Chesselet, M. F. (2002). Synchronous neuronal activity is a signal for axonal sprouting after cortical lesions in the adult. J. Neurosci. 22, 6062-6070.

Carmichael, S. T., Tatsukawa, K. Kornblum, H. I. (2004). Evolution of diaschisis in a focal stroke model. Stroke 35, 758-763.

Chae,J., and Hart, R. (2003). Intramuscular hand neuroprosthesis for chronic stroke survivors. Neurorehabil. Neural. Repair 17, 109-117.

Diamond, M. E., von Heimendahl, M., Knutsen, P. M., Kleinfeld, D., and Ahissar, E. (2008). 'Where' and 'what' Katsman, D., Tsuyuguchi, N., and

damages of the nervous system. Moreover, as electrical stimulation exert angiogenic effects and promotes vascular growth factors preserving the functionality of individual neurons and entire regions, then it is likely that BMIs can be used to extend the time limited Critical Period of enhanced neuroregeneration that opens after stroke.

Controlling both, fields at the penumbra and fields in distant areas will probably require a combination of central and peripheral stimulation. The control of electric fields in the extracellular space achieved by non-invasive TMS/tDCS will be certainly much less precise than with neural implants and the utility of intervention could vary according to the anatomical location of the core area of the stroke. Much more research is required across these lines to evaluate in terms of models the precision that can be achieved by non-invasive modalities. A similar problem is found for peripheral stimulation as we are not yet sure how much selectivity is required in terms of specific nerves or massive stimulation. All these aspects will be better defined once the models of spontaneous recovery are developed.

in the whisker sensorimotor system. Nat. Rev. Neurosci. 9, 601-612.

Floel, A., and Cohen, L. G. (2010). Recovery of function in humans: cortical stimulation and pharmacological treatments after stroke. Neurobiol. Dis 37, 243-251.

Fröhlich, F., and McCormick, D.A. (2010) Endogenous electric fields may guide neocortical network activity. Neuron 67, 129-143.

Furlan, M., Marchal, G., Derlon, J.-M., Baron, J.-C., and Viader, F. (1996). Spontaneous neurological recovery after stroke and the fate of the ischemic penumbra. Ann. Neurol. 40, 216-226.

Grave de Peralta Menendez, R., Gonzalez Andino, S. L., Morand, S., Michel, C. M., and Landis, T. (2000). Imaging the electrical activity of the brain: ELECTRA. Hum Brain Mapp. 9, 1-12.

Gross, C. G. (2000). Neurogenesis in the adult brain: death of a dogma. Nat. Rev. Neurosci. 9, 601-612.

Guadagno, J. V., Calautti, C., and Baron, J.-C. (2003). Progress in imaging stroke: emerging clinical applications. Br. Med. Bull. 65, 145-157.

Hattiangady, B., Rao, M. S., Shetty, G. A. and Shetty, A. K. (2005). Brain-derived neurotrophic factor, phosphorylated cyclic AMP response element binding protein and neuropeptide $\mathrm{Y}$ decline as early as middle age in the dentate gyrus and CA1 and CA3 subfields of the hippocampus. Exp. Neurol. 195, 353-371.

Herrera-Rincon, C., Torets, C., SanchezJimenez, A., Avendano, C., Guillen, P., and Panetsos, F. (2010a). Structural preservation of deafferented cortex induced by electrical stimulation of a sensory peripheral nerve. Conf. Proc. IEEE Eng. Med. Biol. Soc. 5066-5069.
Herrera-Rincon, C., Torets, C., SanchezJimenez, A., Guillen, P., and Panetsos, F. (2010b). "Plasticity in deafferented trigeminal pathway induced by electrical stimulation of peripheral nerve," in Society for Neuroscience Meeting. Program No. 377.2/TT1 2010 Neuroscience Meeting Planner, San Diego, CA.

Jain, N., Florence, S. L., and Kaas, J. H.(1998). Reorganization of somatosensory cortex after nerve and spinal cord injury. News Physiol. Sci. 13, 143-149.

Johansson, B. B. (2011). Current trends in stroke rehabilitation. A review with focus on brain plasticity. Acta Neurol. Scand. 123, 147-159.

Kaas, J. H. (2000).The reorganization of somatosensory and motor cortex after peripheral nerve or spinal cord injury in primates. Prog. Brain Res. 128, 173-179.

Kesar, T. M., Perumal, R., Reisman, D. S., Jancosko, A., Rudolph, K. S., Higginson, J. S., and Binder-Macleod, S. A. (2009). Functional electrical stimulation of ankle plantarflexor and dorsiflexor muscles: effects on poststroke gait. Stroke 40, 3821-3827. Kondziolka, D., Steinberg, G. K., Wechsler, L., Meltzer, C. C., Elder, E., Gebel, J. DeCesare, S., Jovin, T., Zafonte, R., Lebowitz, J., Flickinger, J. C., Tong, D., Marks, M. P., Jamieson, C., Luu, D., Bell-Stephens, T., and Teraoka, J. (2005). Neurotransplantation for patients with subcortical motor stroke: a phase 2 randomized trial. $J$. Neurosurg. 103, 38-45.

Li, W.-L., Yu, S. P., Ogle, M. E., Ding, X. S., and Wei, L. (2008). Enhanced neurogenesis and cell migration following focal ischemia and peripheral 
stimulation in mice. Dev Neurobiol 68 , 1474-1486.

Lo, E. H., Moskowitz, M. A., and Jacobs, T. P. (2005). Exciting, radical, suicidal: how brain cells die after stroke. Stroke 36, 189-192.

Logothetis, N. K., Augath, M., Murayama, Y., Rauch, A., Sultan, F., Goense, J., Oeltermann, A., and Merkle, H. (2010). The effects of electrical microstimulation on cortical signal propagation. Nat. Neurosci. 13, 1283-1291.

Mak, J. N., and Wolpaw, J. R. (2009). Clinical applications of braincomputer interfaces: current state and future prospects. IEEE Rev. Biomed. Eng. 2, 187-199.

Mann, E. O., and Paulsen, O. (2010). Local field potential oscillations as a cortical soliloquy. Neuron 67, 3-5.

McCaig, C. D., Rajnicek, A. M., Song, B., and Zhao, M. (2005). Controlling cell behavior electrically: current views and future potential. Physiol. Rev. 85, 943-978.

McCaig, C. D., Song, B., and Rajnicek, A. M. (2009). Electrical dimensions in cell science. J. Cell. Sci. 122, 4267-4276.

Merzenich, M. M., Kaas, J. H., Wall, J., Sur, M., Nelson, R. J., and Felleman, D. (1983). Progression of changes following median nerve section in the cortical representation of the hand in areas $3 \mathrm{~b}$ and 1 in adult owl and squirrel monkeys. Neuroscience 10, 639-665.

Merzenich, M. M., Recanzone, G. H., Jenkins, W. M., and Grajski, K. A (1990). Adaptive mechanisms in cortical networks underlying cortical contributions to learning and nondeclarative memory. Cold Spring Harb. Symp. Quant. Biol. 55, 873-887.

Molina, C. A., and Saver, J. L. (2005). Extending reperfusion therapy for acute ischemic stroke: emerging pharmacological, mechanical, and imaging strategies. Stroke 36 , 2311-2320.

Murphy, T. H., and Corbett, D. (2009). Plasticity during stroke recovery: from synapse to behaviour. Nat. Rev Neurosci. 10, 861-872.

O’Dell, M.W., Lin, C.-C.D., and Harrison, V. (2009). Stroke rehabilitation: strategies to enhance motor recovery. Annu. Rev. Med. 60, 55-68.

Panetsos, F., and Sanchez-Jimenez, A. (2010). Single unit oscillations in rat trigeminal nuclei and their control by the sensorimotor cortex. Neuroscience 169, 893-905.

Peckham, P. H., and Knutson, J. S. (2005). Functional electrical stimulation for neuromuscular applications. Annu. Rev. Biomed. Eng. 7, 327-360.

Popa-Wagner, A., Carmichael, S. T., Kokaia, Z., Kessler, C., and Walker, L. C. (2007). The response of the aged brain to stroke: too much, too soon? Curr. Neurovasc. Res. 4, 216-227.

Popovic, D. B., Sinkjar, T., and Popovic, M. B. (2009). Electrical stimulation as a means for achieving recovery of function in stroke patients. NeuroRehabilitation 25, 45-58.

Popovic, M. R., Thrasher, T.A.,Zivanovic, V., Takaki, J., and Hajek, V. (2005). Neuroprosthesis for retraining reaching and grasping functions in severe hemiplegic patients. Neuromodulation Technol. Neural Interface 8, 58-72.
Sundelacruz, S., Levin, M., and Kaplan, D. L. (2009). Role of membrane potential in the regulation of cell proliferation and differentiation. Stem Cell Rev. 5 , 231-246.

Tan, J., Widjaja, S., Xu, J., and Shepherd, R. K. (2008). Cochlear implants stimulate activity-dependent creb pathway in the deaf auditory cortex: implications for molecular plasticity induced by neural prosthetic devices. Cereb. Cortex 1799-1813.

Waite, P. M. E., and Tracey, D. J. (1995). "Somatosensory system," in The Rat Nervous System, ed. G. Paxinos (London: Academic Press), 705-724.

Weiss, S. A., and Faber, D. S. (2010). Field effects in the CNS play functional roles. Front Neural Circuits 4:15. doi: 10.3389/fncir.2010.00015

Whitaker, V. R., Cui, L., Miller, S., Yu S. P., and Wei, L. (2006). Whisker stimulation enhances angiogenesis in the barrel cortex following focal ischemia in mice. J. Cereb. Blood Flow Metab. 27, 57-68.

Wong-Riley, M. T. (1989). Cytochrome oxidase: an endogenous metabolic marker for neuronal activity. Trends Neurosci. 12, 94-101.

Woolsey, T. A., Dierker, M. L., and Wann, D. F. (1975). Mouse SmI cortex: qualitative and quantitative classification of golgi-impregnated barrel neurons. Proc. Natl. Acad. Sci. U.S.A. 72 , 2165-2169.

World Health Organization. (2007). World Health Report - 2007, Geneva.

Yan, T., Hui-Chan, C. W.Y., and Li, L. S. W. (2005). Functional electrical stimulation improves motor recovery of the lower extremity and walking ability of subjects with first acute stroke: a randomized placebo-controlled trial. Stroke 36, 80-85.

Yao, L., McCaig, C. D., and Zhao, M. (2009). Electrical signals polarize neuronal organelles, direct neuron migration, and orient cell division. Hippocampus 19, 855-868.

Yao, L., Shanley, L., McCaig, C., and Zhao, M. (2008). Small applied electric fields guide migration of hippocampal neurons. J. Cell. Physiol. $216,527-535$

Conflict of Interest Statement: The authors declare that the research was conducted in the absence of any commercial or financial relationships that could be construed as a potential conflict of interest.

Received: 30 December 2010; accepted: 24 June 2011; published online: 25 July 2011. Citation: Gonzalez Andino SL, HerreraRincon C, Panetsos F and Grave de Peralta $R$ (2011) Combining BMI stimulation and mathematical modeling for acute stroke recovery and neural repair. Front. Neurosci. 5:87. doi: 10.3389/fnins.2011.00087

This article was submitted to Frontiers in Neuroprosthetics, a specialty of Frontiers in Neuroscience.

Copyright (c) 2011 Gonzalez Andino, Herrera-Rincon, Panetsos and Grave de Peralta. This is an open-access article subject to a non-exclusive license between the authors and Frontiers Media SA, which permits use, distribution and reproduction in other forums, provided the original authors and source are credited and other Frontiers conditions are complied with. 